\title{
Surveillance de l'hépatite C au Canada
}

\author{
Payne $\mathrm{E}^{1}$, Totten $\mathrm{S}^{1}$, Archibald $\mathrm{C}^{1}$ \\ ${ }^{1}$ Centre de la lutte contre les maladies transmissibles et les infections, Agence de la santé publique du Canada, Ottawa (Ontario) \\ *Auteure-ressource : elspeth.payne@phac-aspc.gc.ca
}

\section{Résumé}

Contexte : Le virus de l'hépatite $C$ est hautement transmissible et il peut entraîner une maladie chronique du foie. II constitue toujours une préoccupation majeure en matière de santé publique au Canada.

Objectif : Décrire les tendances de surveillance des cas déclarés du virus de l'hépatite C (VHC) au Canada, par groupe d'âge et par sexe, de 1991 à 2012.

Méthodologie : On a compilé à l'échelle nationale les cas d'infection par le VHC déclarés au Système canadien de surveillance des maladies à déclaration obligatoire. Étant donné que la plupart des cas signalés sont déclarés sans distinction du stade de l'infection par le VHC chronique ou aiguë, les résultats présentés sont fondés sur le nombre total de cas d'infection par le VHC. Les tendances au fil du temps sont fournies de 1991 à 2012, avec un examen plus détaillé des profils d'âge et de sexe de 2005 à 2012.

Résultats : Le taux des cas déclarés d'infection par le VHC a fortement augmenté de 1991 (l'année où elle d'abord été à déclaration obligatoire) à 1998, année à laquelle on a observé le plus haut taux global de 66,9 cas pour 100000 habitants. De 1998 à 2012, les taux de cas déclarés ont diminué chez les deux sexes, mais sont restés systématiquement plus élevés chez les hommes que chez les femmes. En 2012, le taux global de cas déclarés d'infection par le VHC était de 29,3 cas pour 100000 habitants. Dans les groupes d'âge plus jeunes, les taux chez les femmes étaient légèrement plus élevés, tandis que les hommes dans les groupes plus âgés (plus de 30 ans) présentaient des taux considérablement plus élevés.

Conclusion : Le présent résumé de surveillance présente les tendances à plus long terme des cas déclarés et des taux correspondants d'infection par le VHC au Canada à l'aide des données de surveillance nationale. Le Canada continue de présenter une tendance à la baisse dans les taux de VHC; toutefois, le fardeau de l'infection continuera d'augmenter au fur et à mesure que les personnes infectées développent une maladie grave.

\section{Introduction}

Le virus de l'hépatite $C$ est hautement transmissible; il se transmet par contact avec du sang contaminé.

L'infection par le virus de l'hépatite $C$ peut entraîner une maladie hépatique chronique et elle touche environ $3 \%$ de la population mondiale (1). À l'échelle mondiale, environ 130 à 150 millions de personnes sont atteints d'une infection chronique par le virus de l'hépatite $\mathrm{C}(\mathrm{VHC})$ et 350000 à 500000 personnes meurent chaque année de maladies liées au VHC (1). L'Enquête sur les mesures de la santé dans la collectivité a récemment estimé la séroprévalence des anticorps anti-VHC chez la population générale au Canada à 0,5\% (1), tandis que des modèles épidémiologiques et mathématiques intégrant des données issues de populations à risque élevé ont permis d'estimer la séroprévalence des anticorps anti-VHC à environ 0,96 \% (1). En 2011, l'infection aiguë et l'infection chronique par le VHC ont été signalées comme cause sous-jacente de 35 et 346 décès, respectivement, au Canada (5). II se peut que ces chiffres soient sous-estimés, des décès attribuables à l'infection chronique par le VHC ayant pu être codifiés comme imputables à une cause plus immédiate, par exemple un carcinome hépatocellulaire ou une cirrhose. 
Le VHC a été identifié pour la première fois en 1989, ce qui a facilité l'élaboration de méthodes de dépistage par l'amplification de l'acide nucléique et des anticorps anti-VHC qui ont été mises en œuvre pour le dépistage de la réserve de sang au Canada en 1990. Avant cette découverte, des milliers de personnes ont été infectées par le VHC après avoir reçu des transfusions de composants ou de produits sanguins. L'introduction du dépistage universel de la réserve de sang a beaucoup amélioré la qualité de la réserve de sang du Canada et a pratiquement éliminé ce risque de transmission (6).

Cependant, certaines populations continuent de se trouver à un risque plus élevé d'infection par le VHC. La transmission du VHC chez les utilisateurs de drogues injectables est maintenant le facteur qui contribue le plus aux taux globaux d'infection par le VHC; la majorité des infections récentes par le VHC au Canada sont attribuables au partage du matériel de préparation et d'injection de drogues (7).

Les voies de transmission du VHC moins fréquentes comprennent le contact avec du sang contaminé par le partage d'instruments pointus, de matériel d'hygiène personnelle ( $p$. ex. les rasoirs, les brosses à dents, les ciseaux et les coupe-ongles) et la transmission verticale de la mère à l'enfant $(8,9)$. Même si la transmission sexuelle du VHC est rare, ce mode de transmission a été observé chez les hommes ayant des relations sexuelles avec d'autres hommes infectés par le VIH (10).

Le VHC représente toujours une préoccupation importante en matière de santé publique et a été classé le premier parmi 51 pathogènes pour sa contribution relative à l'ensemble du fardeau des maladies infectieuses en Ontario (11). L'hépatite $C$ n'est pas évitable par la vaccination et même si certaines personnes éliminent leur infection et se rétablissent spontanément, l'hépatite $C$ se transforme en infection chronique avec des conséquences potentiellement mortelles chez $85 \%$ des personnes touchées (12). Le fardeau des soins de santé présenté par les cas existants qui entraînent de plus lourdes séquelles continue de s'intensifier.

L'objectif de cette analyse était de décrire les tendances des cas d'hépatite $C$ déclarés au Système canadien de surveillance des maladies à déclaration obligatoire (SCSMDO) de 1991 à 2012, avec un examen plus détaillé des profils de groupes d'âge et de sexe de 2005 à 2012.

\section{Méthodologie}

\section{Sources des données}

Les données sur les cas d'infection par le VHC sont déclarées au SCSMDO par les ministères provinciaux et territoriaux de la Santé, lesquels obtiennent ces données des autorités sanitaires locales et régionales. L'infection par le VHC est à déclaration obligatoire à l'échelle nationale depuis 1991. La surveillance des cas d'hépatite C était graduelle au fil du temps par les provinces et les territoires, et l'ensemble des provinces et territoires déclaraient les cas en 1999. Les données du Nunavut de 2007 à 2012 n'étaient pas disponibles pour cette analyse.

La définition des cas d'infection par le VHC utilisée à des fins de surveillance nationale a évolué depuis 1991 (13), avec des révisions en 1999 (14), 2008 (15) et 2011 (Mise à jour inédite de 2011. Agence de la santé publique du Canada. Définitions de cas des maladies transmissibles faisant l'objet d'une surveillance nationale). La plupart des provinces et des territoires confirment les cas en utilisant le dépistage des anticorps anti-VHC et ne distinguent actuellement pas l'état des cas déclarés d'infection par le VHC. Par conséquent, les cas d'infection aiguë et chronique par le VHC ont été combinés aux fins d'analyse.

\section{Analyse}

Une analyse descriptive de l'infection à VHC par année, par groupe d'âge et par sexe a été menée à partir des données déclarées au SCSMDO. Les analyses incluaient tous les cas d'hépatite $C$ déclarés au SCSMDO et les taux sont exprimés pour 100000 habitants. Étant donné que l'hépatite $C$ n'a pas été déclarée par l'ensemble des provinces et des territoires au cours de certaines périodes, les taux nationaux pour chaque année ont été calculés avec des dénominateurs ajustés de façon à inclure uniquement les provinces et territoires pour lesquels les données étaient accessibles. Les caractéristiques démographiques d'âge et de sexe ont été examinées pour les 
cas d'hépatite $\mathrm{C}$ déclarés en 2012 afin de fournir un aperçu plus détaillé des données accessibles les plus récentes.

Les données sur la population qui ont servi à calculer les taux ont été obtenues auprès de Statistique Canada (16). Les estimations suivantes ont été utilisées : estimations intercensitaires définitives pour 2005; estimations postcensitaires définitives pour 2006; estimations intercensitaires définitives pour 2007-2010; estimations postcensitaires définitives pour 2011; et estimations postcensitaires mises à jour pour 2012. Les taux, les pourcentages et le pourcentage de variation des taux ont été calculés à partir de valeurs non arrondies; par conséquent, les résultats présentés peuvent différer par rapport aux calculs effectués à partir de valeurs arrondies. Étant donné que les données sur les dénominateurs de la population ont été mises à jour, les taux déclarés peuvent différer de ceux des rapports précédents.

\section{Résultats}

\section{Tendances à long terme (1991-2012)}

Entre 1991 et 1998, on a observé une augmentation globale du taux de cas déclarés d'hépatite C. En 1991, 912 cas ont été déclarés, ce qui correspond à un taux de 5,2 cas pour 100000 habitants. En 1998, ce taux avait augmenté de plus de $1000 \%$, atteignant 19379 cas et un taux de 66,9 cas pour 100000 habitants. Tout au long de cette période, les taux de cas déclarés d'hépatite $C$ ont été systématiquement plus élevés chez les hommes que chez les femmes (Figure 1).

De 1998 à 2012, le taux de cas déclarés d'hépatite $C$ a constamment diminué chez les hommes comme les femmes. En 2012, 10180 cas d'hépatite $C$ ont été déclarés, ce qui correspond à un taux de 29,3 cas pour 100000 habitants et à une diminution de 56,2 \% par rapport à 1998. Même si le taux des cas déclarés d'hépatite $C$ était systématiquement plus élevé chez les hommes que chez les femmes au cours de cette période, l'écart entre les deux sexes s'est amenuisé au fil du temps. Chez les hommes, les taux ont diminué de 58,6\%, passant de 88,9 à 36,8 cas pour 100000 habitants; chez les femmes, les taux ont diminué de $52,3 \%$, passant de 45,0 à 21,5 cas pour 100000 habitants (Figure 1).

Figure 1 : Taux de cas déclarés d'hépatite C au Canada ${ }^{1}$ selon le sexe, SCSMDO², 1991-2012

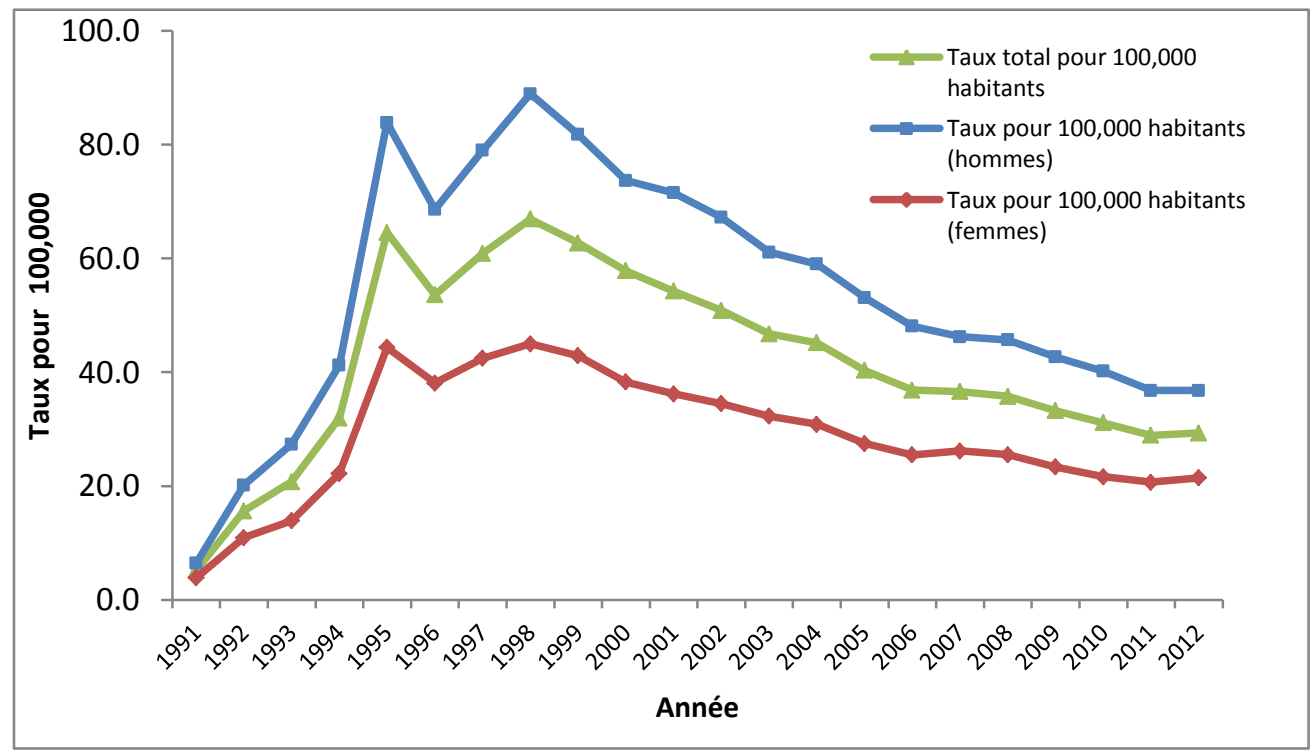

${ }^{1}$ Comprend l'Île-du-Prince-Édouard, l'Ontario, la Saskatchewan, l'Alberta, la Colombie-Britannique, 1991-2012; Terre-Neuve-et-Labrador, le NouveauBrunswick, Territoires du Nord-Ouest, 1993-2012; Yukon, 1994-2012; Nouvelle-Écosse, Québec, 1996-2012; Manitoba, 1999-2012; Nunavut, 1999-2006. Pour le calcul des taux, les dénominateurs de la population ont été ajustés pour inclure uniquement les provinces et territoires pour lesquels des données étaient accessibles pour une année donnée.

${ }^{2} \mathrm{SCSMDO}=$ Système canadien de surveillance des maladies à déclaration obligatoire 
Récentes tendances selon le groupe d'âge et le sexe (de 2005 à 2012)

Entre 2005 et 2012, on a observé des baisses des taux chez les hommes de tous les groupes d'âge, à l'exception de ceux âgés de 60 ans et plus, qui ont connu une légère augmentation du taux qui est passé de 21,8 à 26,3 cas pour 100000 habitants. Chez les hommes âgés de moins de 15 ans, les taux d'infection par le VHC étaient moins de 1,0 cas pour 100000 habitants pour toutes les années. En 2005, les hommes âgés de 40 à 59 ans détenaient le taux le plus élevé de cas déclarés d'infection aiguë par le VHC, soit 101,0 cas pour 100000 habitants.

En 2012, ce taux avait diminué jusqu'à 64,4 cas pour 100000 habitants. Une importante diminution du taux a également été observée chez les hommes de 30 à 39 ans; de 2005 à 2012, les taux d'infection par le VHC ont diminué, passant de 83,9 à 51,7 cas pour 100000 habitants (Figure 2).

Figure 2 : Taux de cas déclarés d'hépatite $C$ chez les hommes au Canada ${ }^{1}$ par groupe d'âge, SCSMDO², 2005-2012

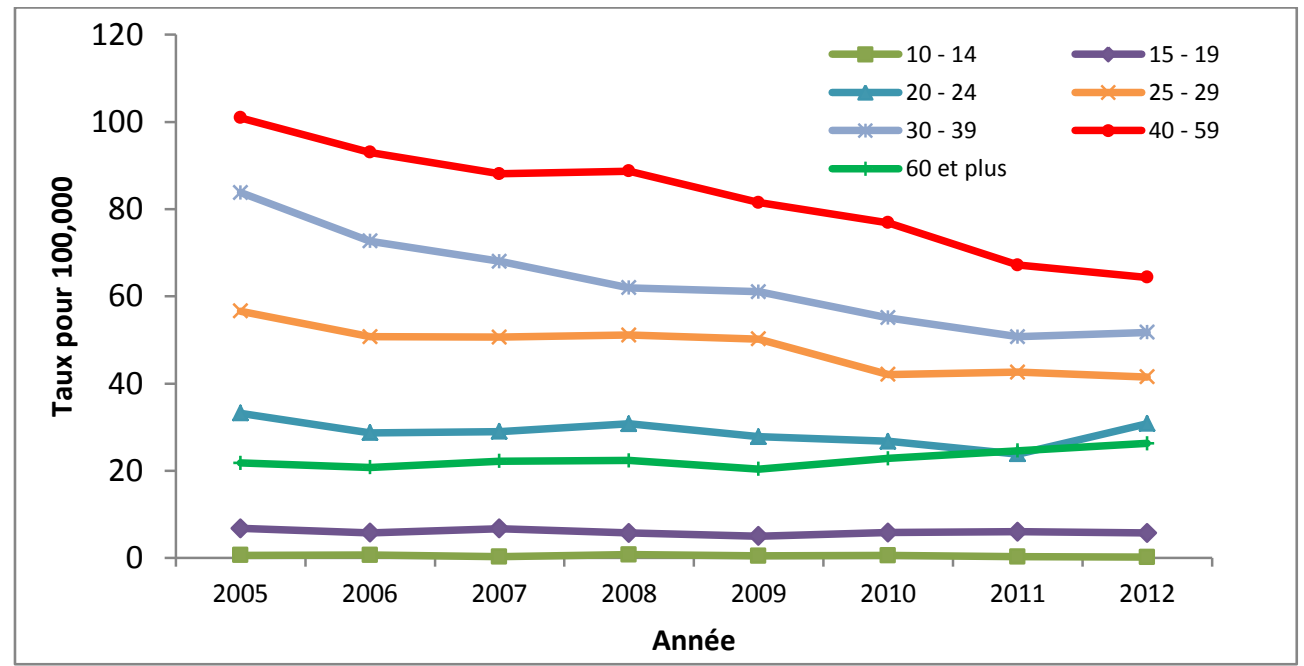

\footnotetext{
${ }^{1}$ Au moment de l'analyse, les données sur l'hépatite C de 2007 à 2012 n'étaient pas disponibles pour le Nunavut. La population a été supprimée du dénominateur utilisé pour le calcul du taux.

${ }^{2} \mathrm{SCSMDO}=$ Système canadien de surveillance des maladies à déclaration obligatoire
}

Au cours de la même période, des baisses des taux ont été observées chez les femmes, à l'exception de celles dans le groupe d'âge de 25 à 29 ans, qui ont connu une augmentation du taux de 35,6 à 38,2 cas pour 100000 habitants. La diminution de taux la plus importante a été observée chez les femmes de 10 à 14 ans, passant de 1,2 à 0,3 cas pour 100000 habitants. Cette diminution est en grande partie le reflet de la faible numération du VHC au sein de ce groupe d'âge et de l'instabilité des taux qui en résulte. À l'exclusion des femmes de 10 à 14 ans, la plus forte diminution de taux a été observée chez les femmes de 30 à 39 ans, de 45,6 à 32,3 cas pour 100000 habitants (Figure 3 ). 
Figure 3 : Taux de cas déclarés d'hépatite C chez les femmes au Canada ${ }^{1}$ par groupe d'âge, SCSMDO ${ }^{2}$, 2005-2012

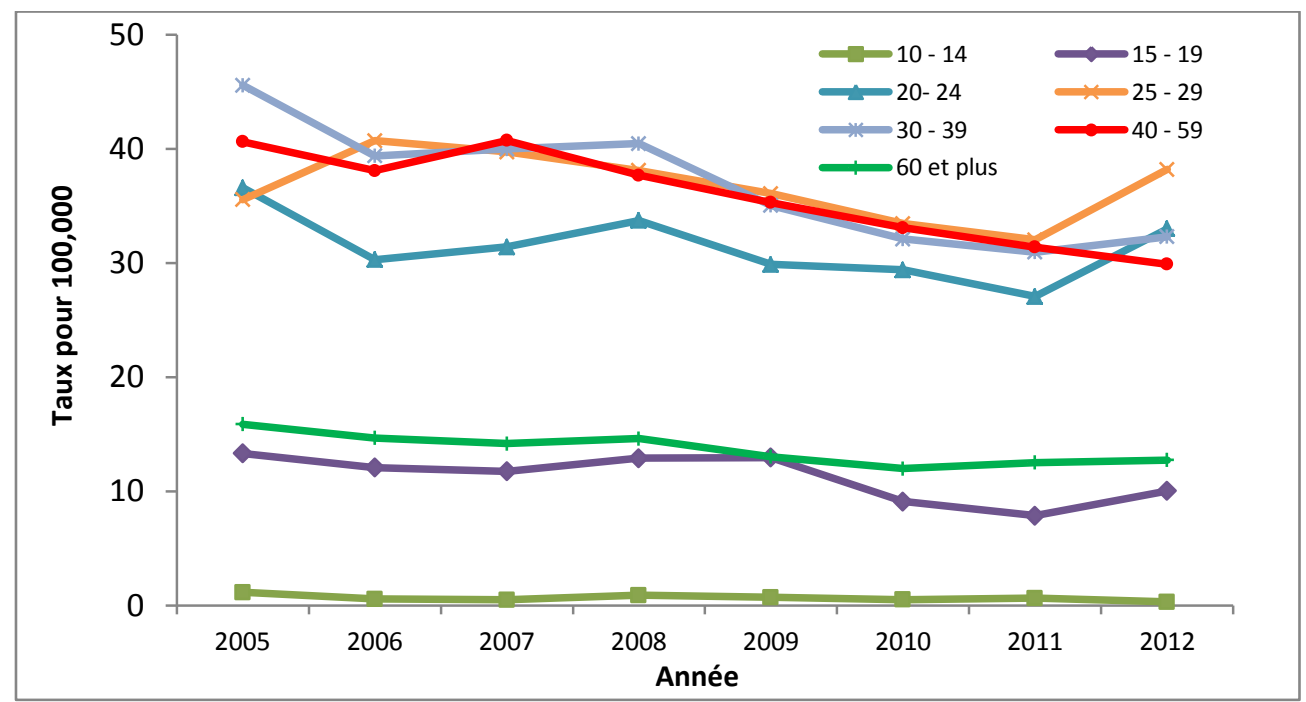

${ }^{1}$ Au moment de l'analyse, les données sur l'hépatite C de 2007 à 2012 n'étaient pas disponibles pour le Nunavut. La population a été supprimée du dénominateur utilisé pour le calcul du taux.

${ }^{2} \mathrm{SCSMDO}=$ Système canadien de surveillance des maladies à déclaration obligatoire

En 2012, le taux le plus élevé de cas d'hépatite $C$ a été observé chez les hommes de 40 à 59 ans, suivis des hommes de 30 à 39 ans (Figure 4). Pour les deux sexes, les taux étaient plus élevés chez les personnes de plus de 19 ans. Les différences entre les taux des hommes et des femmes étaient plus prononcées dans les groupes plus âgés (de 30 ans et plus), et les hommes présentaient des taux considérablement plus élevés.

Figure 4 : Taux de cas déclarés d'hépatite $\mathrm{C}$ au $\mathrm{Canada}^{1}$ par groupe d'âge et par sexe, SCSMDO, 2012

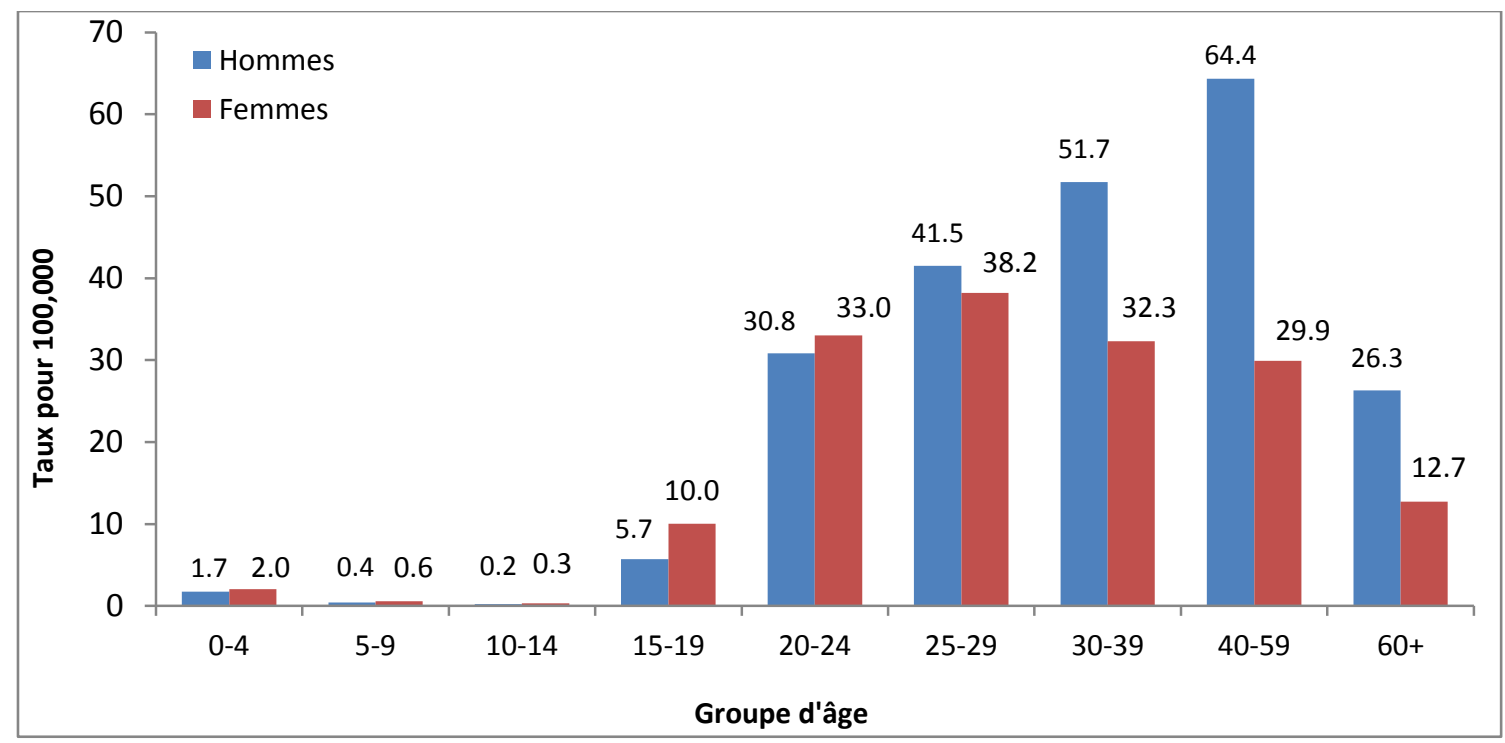

\footnotetext{
${ }^{1}$ Au moment de l'analyse, les données sur l'hépatite C de 2012 n'étaient pas disponibles pour le Nunavut, et cette population a été supprimée du calcul du taux.

${ }^{2}$ SCSMDO $=$ Système canadien de surveillance des maladies à déclaration obligatoire
} 


\section{Discussion}

Cette analyse fournit les données les plus à jour actuellement disponibles sur les cas déclarés et les taux correspondants de cas d'infection par le VHC au Canada.

De 1991 à 1998, le taux de cas déclarés d'hépatite $\mathrm{C}$ au Canada a augmenté de façon constante au fur à mesure que les personnes infectées par la réserve de sang étaient déterminées par les procédures de retraçage des donneurs et des receveurs (17). Une sensibilisation croissante au risque de transmission du VHC à la lumière de l'enquête à l'échelle nationale menée par la Commission Krever (18) et les reportages connexes des médias peuvent également avoir eu une incidence sur les taux de dépistage du VHC, ce qui contribue à une hausse du nombre de cas déclarés au cours de cette période. La mise en œuvre de la surveillance du VHC par les provinces et les territoires dans l'ensemble du Canada ne devrait pas avoir une incidence considérable sur l'augmentation des taux au cours de cette période, étant donné que les taux ont été calculés à l'aide de dénominateurs ajustés.

Depuis 1998, les taux de cas déclarés d'infection par le VHC ont diminué de 56,2 \%, même si certaines populations sont toujours à risque. Parmi les nouveaux cas d'infection par le VHC possédant des renseignements en matière de facteurs de risque connus, $61 \%$ avaient déclaré des antécédents d'utilisation de drogues injectables (19). I-Track, système de surveillance comportementale et biologique à l'échelle nationale qui assure le suivi du $\mathrm{VIH}$ et de l'hépatite $\mathrm{C}$ et des comportements à risque associés parmi les utilisateurs de drogues injectables au Canada, a permis de révéler que l'exposition à vie à l'infection par le VHC (tel qu'il a été mesuré par la présence d'anticorps anti-VHC dans un échantillon de gouttes de sang séchées) était de $68 \%$ au cours de la phase 3 de la collecte de données effectuée de 2010 à 2012 (20). Au cours de la phase 3 de l-Track, 68,2\% des participants étaient de sexe masculin (20), ce qui indique qu'une plus grande proportion de personnes qui utilisent des drogues injectables sont des hommes et qui explique potentiellement pourquoi les hommes représentent toujours une plus grande proportion des cas déclarés d'infection par le VHC. Toutefois, les différences entre les taux de cas déclarés d'infection par le VHC chez les hommes et les femmes se sont amenuisées depuis 2005; les femmes sont plus susceptibles de recevoir de l'aide pour l'utilisation de drogues injectables ou de se trouver dans des partenariats sexuels qui reposent sur l'échange de drogues et présentent un risque plus élevé de partage de matériel de consommation de drogues (21). Les taux variables chez les hommes et les femmes peuvent également être le reflet des différents comportements liés aux tests sérologiques. Les femmes sont plus susceptibles d'avoir recours à des soins de santé et de subir un test de dépistage (22), menant à une augmentation des taux de cas déclarés d'infection par le VHC.

Une autre source de cas d'infection par le VHC peut être l'immigration au Canada en provenance de pays où l'hépatite $C$ est endémique, en particulier des régions où des précautions universelles pour prévenir la transmission des infections transmissibles par le sang ne sont pas systématiquement mises en œuvre (23). Les contacts familiaux, la transmission verticale et la transmission sexuelle sont moins courants au Canada et peu susceptibles de contribuer à un nombre important de cas du total national.

Une analyse des effets de cohorte chez les cas déclarés d'infection par le VHC a révélé que les personnes nées entre 1946 et 1965 ont contribué à plus de la moitié de tous les cas d'infection par le VHC déclarés entre 1991 et 2010 au Canada (24). Même si le taux de cas déclarés au Canada semble être en baisse, le nombre de personnes infectées depuis des décennies et qui connaissent des séquelles est susceptible d'augmenter au fil du temps à mesure que les personnes atteignent les stades plus graves d'évolution de la maladie (25). En outre, les cas non diagnostiqués représentent un fardeau futur inconnu de la maladie (25).

Même s'il n'existe aucun vaccin, il existe des traitements pour l'infection par le VHC. Auparavant, le traitement était limité à l'interféron pégylé- $\alpha$ en combinaison avec la ribavirine (26). Cependant, des antiviraux à action directe (AAD) très efficaces ont récemment été approuvés par Santé Canada $(27,28)$ et d'autres encore sont en cours d'élaboration clinique (29). Au fil du temps, une augmentation de la disponibilité de nouveaux traitements et de la mise en œuvre d'autres interventions en santé publique (30) peut réduire la transmission et avoir une incidence sur les taux de nouveaux cas déclarés. 


\section{Limites}

L'interprétation de ces résultats doit tenir compte des diverses limites des données. Tout d'abord, la définition de cas d'infection par le VHC utilisée à des fins de surveillance nationale et de protocoles de production de rapports utilisés par les provinces et les territoires a évolué depuis 1991, ce qui pourrait avoir eu une influence sur la déclaration de cas au fil du temps. La définition de cas a systématiquement permis de réaliser des tests de confirmation à l'aide d'un essai immunoenzymatique (EIA) d'un deuxième fabricant. Les plus faibles sensibilité et spécificité de la première et de la deuxième génération de ces essais de VHC ont probablement donné lieu à une proportion inconnue de cas faux positifs d'infection par le VHC (31).

On ignore dans quelle mesure ces données reflètent l'incidence réelle du VHC. Les personnes atteintes d'une infection aiguë par le VHC sont souvent asymptomatiques et, par conséquent, elles pourraient ne pas avoir subi un test ou reçu un diagnostic. Selon les résultats de l'Enquête sur les mesures de la santé dans la collectivité, seulement $30 \%$ des répondants canadiens dont les tests avaient révélé la présence actuelle d'une infection par le $\mathrm{VHC}$ ont indiqué qu'ils avaient reçu un diagnostic d'infection par le VHC (3). De plus, en raison de la longue durée de l'infection, il est possible qu'une personne ait subi un test de dépistage et reçu un diagnostic dans plus d'une province ou d'un territoire au fil du temps et, par conséquent, qu'elle ait été comptée plus d'une fois dans l'ensemble de données du SCSMDO.

Les renseignements sur le caractère aigu ou chronique des cas déclarés d'infection par le VHC n'étaient pas accessibles pour la plupart des provinces et des territoires. Par conséquent, les résultats ne peuvent pas mettre en lumière les tendances actuelles possibles en matière de transmission ou le fardeau potentiel de l'infection par le VHC au Canada.

Enfin, les données ont été analysées uniquement en fonction de l'âge et du sexe. Pour le moment, le Système canadien de surveillance des maladies à déclaration obligatoire ne dispose d'aucun autre élément de données permettant d'expliquer les tendances observées. Par conséquent, il est impossible d'établir avec certitude la proportion de cas déclarés d'infections par le VHC attribuables à la transmission par l'utilisation de drogues injectables ou à d'autres facteurs de risque.

\section{Conclusion}

À la suite de l'augmentation initiale du nombre de cas au cours d'une période de hausse du dépistage, la tendance à la baisse du taux de nouveaux cas déclarés d'infections par le VHC s'est maintenue au Canada. Malgré d'importantes limites, ces résultats contribuent à notre compréhension du VHC au Canada. La surveillance, appuyée par des recherches axées sur l'examen de facteurs ayant une incidence sur les tendances observées, peut contribuer à la mise au point d'interventions adaptées à la réalité de l'infection par le VHC au Canada.

\section{Remerciements}

Les auteurs tiennent à remercier les fournisseurs de soins de santé, les responsables de la santé publique, les laboratoires et les provinces et les territoires qui ont participé aux activités de déclaration systématique de I'hépatite C. Nous désirons également remercier Rachel MacLean pour sa contribution aux activités de surveillance de l'hépatite $C$ de l'Agence de la santé publique du Canada.

\section{Conflit d'intérêts}

Aucun

\section{Financement}

Le financement de ce projet a été assuré par l'Agence de la santé publique du Canada. 


\section{Références}

(1) Mohd Hanafiah K, Groeger J, Flaxman AD, Wiersma ST. Global epidemiology of Hepatitis C virus infection: new estimates of age-specific antibody to HCV seroprevalence. Hepatology 2013; Apr;57(4):1333-1342.

(2) Organisation mondiale de la santé. Hépatite $C$ (Aide-mémoire Numéro 164). http://www.who.int/mediacentre/factsheets/fs164/fr/

(3) Rotermann M, Langlois K, Andonov A, Trubnikov M. Séroprévalence des infections par le virus de l'hépatite B et par le virus de l'hépatite C : résultats de l'Enquête canadienne sur les mesures de la santé, 2007 à 2009 et 2009 à 2011. L'Enquête canadienne sur les mesures de la santé. Rapport sur la santé 2013; 24 (11) : 3-14.

(4) Trubnikov M, Yan P, Archibald C. Estimation de la prévalence de l'infection par le virus de l'hépatite C au Canada, 2011. Relevé des maladies transmissibles au Canada 2014;40-19; 429-436.

(5) Statistique Canada, Statistique de l'état civil du Canada. Décès, selon la cause, Chapitre I: Certaines maladies infectieuses et parasitaires (A00 à B99), le groupe d'âge et le sexe, Canada. http://www5.statcan.gc.ca/cansim/a05?lang=fra\&id=1020521

(6) O'Brien SF, Yi QL, Fan W, Scalia V, Fearon MA, Allain JP. Current incidence and residual risk of HIV, HBV and HCV at Canadian Blood Services. Vox Sang. 2012; Jul;103(1):83-86.

(7) Delage G, Infante-Rivard C, Chiavetta JA, Willems B, Pi D, Fast M. Risk factors for acquisition of Hepatitis C virus infection in blood donors: Results of a case-control study. Gastroenterology. 1999; Apr;116(4):893-899.

(8) Bevilacqua E, Fabris A, Floreano P, Pembrey L, Newell ML, Tovo PA, et al. Genetic factors in mother-to-child transmission of HCV infection. Virology. 2009; Jul 20;390(1):64-70.

(9) Ngo-Giang-Huong N, Jourdain G, Sirirungsi W, Decker L, Khamduang W, Le Coeur S, et al. Human immunodeficiency virus-Hepatitis $\mathrm{C}$ virus co-infection in pregnant women and perinatal transmission to infants in Thailand. Int $\mathrm{J}$ Infect Dis. 2010; Jul;14(7):e602-7.

(10) Myers T, Allman D, Xu K, Remis RS, Aguinaldo J, Burchell A, et al. The prevalence and correlates of Hepatitis C virus (HCV) infection and HCV-HIV co-infection in a community sample of gay and bisexual men. Int J Infect Dis. 2009; Nov;13(6):730-739.

(11) Kwong J, Crowcroft N, Campitelli M, Ratnasingham S, Daneman N, Deeks S, et al. Ontario Burden of Infectious Disease Study (ONBOIDS): An OAHPP/ICES Report. Toronto: Ontario Agency for Health Protection and Promotion, Institute for Clinical Evaluative Sciences; 2010.

(12) Seeff LB. The history of the "natural history" of Hepatitis C (1968-2009). Liver Int. 2009; Jan;29 Suppl 1:89-99.

(13) Santé Canada. Programme canadien de surveillance des maladies transmissibles: définitions de cas et méthodes de surveillance particulières à chaque maladie : H12-21/17-S3. Relevé Hebdomadaire Médical Canada 1991; 17S3

(14) Santé Canada. Définitions de cas des maladies faisant l'objet d'une surveillance nationale - 2000: H12-21-3/26-3F. Relevé des maladies transmissibles au Canada 2000; 26S3:69.

(15) Agence de la santé publique du Canada. Supplément- Définitions nosologiques des maladies transmissibles faisant l'objet d'une surveillance nationale-2009. Relevé des maladies transmissibles au Canada 2009; 35 (Supplément 2).

(16) Statistique Canada, Division de la démographie, Section des estimations démographiques. Estimation de la population en juillet. 2013.

(17) Norris S. Publications de recherche de la bibliothèque du Parlement. L'approvisionnement en sang du Canada dix ans après la Commission Krever. Bibliothèque du Parlement 2008; PRB 08-14E.

(18) Krever H. Commission d'enquête sur l'approvisionnement en sang au Canada, Rapport final. Ottawa : La Commission 1997;CP32-62/3-1997F-PDF.

(19) Agence de la santé publique du Canada, Section de la surveillance et de l'épidémiologie des ITS et de l'hépatite C. Épidémiologie de l'infection aiguë par le virus de l'hépatite $\mathrm{C}$ au Canada. Résultats du Système de surveillance accrue des souches de l'hépatite (SSASH), 2009.

(20) Agence de la santé publique du Canada, Principales conclusions émanant d'un système national de surveillance amélioré du VIH : 2010-2012. Division de l'épidémilologie et de surveillance, Centre de la lutte contre les maladies transmissibles et les infections : 2014

(21) Tompkins C, Sheard L, Wright N, Jones L, Howes N. Exchange, deceit, risk, harm: The consequences for women of receiving injections from other drug users. Drugs: education, prevention and policy. 2006; 13:281-297.

(22) Galdas PM, Cheater F, Marshall P. Men and health help-seeking behaviour: Literature review. J Adv Nurs. 2005; Mar;49(6):616-623. 
(23) Ellison LF, Wilkins K. Étude : Tendances de la prévalence du cancer au Canada. Rapport sur la santé2012; 23(1):7-16.

(24) Trubnikov M, Yan P, Njihia J, Archibald C. Identifying and describing a cohort effect in the national database of reported cases of Hepatitis C virus infection in Canada (1991-2010): An age-period-cohort analysid. CMAJ Open in press 2014.

(25) Remis RS. Modélisation de l'incidence et de la prévalence de l'C et de ses séquelles au Canada, 2007. Ottawa : Agence de la santé publique du Canada; 2007.

(26) Sherman M, Shafran S, Burak K, Doucette K, Wong W, Girgrah N, et al. Management of chronic Hepatitis C: Consensus guidelines. Can J Gastroenterol. 2007; Jun;21 Suppl C:25C-34C.

(27) Santé Canada. Médicaments et produits de santé : Galexos. 2014. http://www.hc-sc.gc.ca/dhp-mps/prodpharma/sbdsmd/drug-med/sbd_smd_2014_galexos_164021-fr.php.

(28) Santé Canada. Médicaments et produits de santé : Sovaldi. 2014. http://www.hc-sc.gc.ca/dhp-mps/prodpharma/sbdsmd/drug-med/sbd_smd_2014_sovaldi_165043-fra.php

(29) Kiser JJ, Flexner C. Direct-acting antiviral agents for Hepatitis C virus infection. Annu Rev Pharmacol Toxicol. 2013; 53:427-429.

(30) Hagan H, Pouget ER, Des Jarlais DC. A systematic review and meta-analysis of interventions to prevent Hepatitis C virus infection in people who inject drugs. JID. 2011; 204:74-83.

(31) Kesli R. Evaluation of assay methods and false positive results in the laboratory diagnosis of Hepatitis $\mathrm{C}$ virus infection. Archives of Clinical Microbiology. 2011; 2(4):1-4. 